\title{
Surgical and transcatheter (Amplatzer) closure of atrial septal defects: a prospective comparison of results and cost
}

\author{
J D R Thomson, E H Aburawi, K G Watterson, C Van Doorn, J L Gibbs
}

See end of article for authors' affiliations

\section{Correspondence to:} Dr J D R Thomson, Department of Paediatric Cardiology, E floor, Jubilee Wing, Leeds General Infirmary, Great George St, Leeds LS1 3EX, UK; john.thomson@lineone.net Accepted 7 November 2001
Heart 2002;87:466-469

\begin{abstract}
Objective: To compare effectiveness, complications, and cost of Amplatzer with surgical atrial septal defect (ASD) closure.

Design: Prospective study.

Setting: Tertiary cardiac referral centre.

Patients: 43 consecutive patients (excluding non-UK residents) aged between 2.1 and 56.8 years (median 7) undergoing ASD closure.

Main outcome measures: Procedural success, complications, regression of right ventricular dilatation (up to one year postprocedure), cost, inpatient stay, and home convalescent time.

Results: Amplatzer ASD closure was successful in 24 of 27 (89\%) patients. Surgical closure was successful in all 19 cases. Cardiac complications affecting management occurred in three $(11 \%)$ of the Amplatzer group (two procedural failures, one device embolisation) and 4 of $19(21 \%)$ surgical patients (one pericardial pain, one global pericardial effusion requiring drainage, and one patient with anaemia requiring haematinics in addition to an incidental pericardial effusion and one further incidental pericardial effusion) ( $p=N S$ ). There were complications that did not affect management in a further 5 of 19 surgical patients. There was no significant difference in regression of right ventricular dilatation by six months postprocedure (median right ventricular end diastolic diameter decrease: Amplatzer group 17.5\%, surgical group 15.1\%; median cardiothoracic ratio decrease: Amplatzer $7.9 \%$, surgical $7.5 \%$ ). Both hospital stay and home convalescent times were significantly shorter after Amplatzer closure (median hospital stay: Amplatzer one day, surgery six days; median convalescent time: Amplatzer two weeks, surgery 5.5 weeks). Median cost was similar for both groups (Amplatzer $£ 5375$, surgical $£ 5412$ ).

Conclusions: Amplatzer ASD closure has a lower chance of success with a single procedure than surgery. Overall, there were more complications in the surgical group but the majority of these were minor and did not require any change in management. Resolution of right ventricular dilatation over the study period was similar for both techniques. Time spent in hospital and away from work or school was shorter for the Amplatzer group. The cost of both techniques was similar.
\end{abstract}

$\mathrm{N}$ on-surgical secundum atrial septal defect (ASD) closure was first described in 1976 by King and colleagues ${ }^{1}$ and is now a widely available form of treatment for suitable lesions. ${ }^{2-5}$ Despite the increasing number of transcatheter closures there is little information comparing the effectiveness, cost, and complications of the technique with conventional surgery.

\section{PATIENTS AND METHODS}

Between May 1998 and February 2000, 43 consecutive patients who were resident in the UK (median age 7 years, range $2.1-56.8$ years) underwent closure of a haemodynamically significant secundum ASD. Patients were assessed by a standard echocardiographic protocol. All had full transthoracic examinations with a Sonos 2000 ultrasound system (Hewlett Packard, Palo Alto, California, USA) with a transducer appropriate to size and body weight (2.0$7.5 \mathrm{MHz}$ ). All patients had echocardiographic evidence of right ventricular dilatation.

Suitability for non-surgical closure was assessed according to the technique described by Reddy and colleagues. ${ }^{6}$ The diameter of the ASD was measured as were distances to the mitral valve, coronary sinus, pulmonary veins, superior vena cava, and inferior vena cava. Patients were not randomised to treatment groups. Those with suitable margins on transthoracic echocardiography were offered non-surgical closure.
The surgical group consisted of patients with deficient margins on two dimensional transthoracic echocardiography, patients in whom attempted non-surgical closure had failed, and patients with defects suitable for non-surgical closure but who opted for surgery. Subjects were prospectively followed up for up to one year.

\section{Non-surgical closure}

All procedures were performed under general anaesthesia. A detailed transoesophageal echocardiogram was obtained using the Sonos 2000 ultrasound system. No patients were found to have unsuitable margins on transoesophageal echocardiogram.

Cardiac catheterisation was carried out through the femoral vein. Pulmonary angiography was performed to exclude anomalous pulmonary venous return. Balloon sizing was determined using either a Meditech (Boston Scientific, Natick, Massachusetts, USA), Robicsek (BVM Medical, Earl Shelton, UK) or Fogarty (Baxter, Deerfield, Illinois, USA) balloon catheter. An Amplatzer ASD closure device of a size equal to the stretched defect diameter was deployed.

Abbreviations: ASD, atrial septal defect; RVEDD, right ventricular end diastolic diameter 
Table 1 Characteristics and outcomes in patients according to treatment

\begin{tabular}{llll}
\hline & Amplatzer group & Surgical group & p Value \\
\hline Number & 24 & 19 & NS \\
Median age (years, range) & $9.7(2.1-44.6)$ & $5.5(2.7-15.2)$ & 0.01 \\
Median atrial septal defect size (mm, range) & $13(9-30)$ & $19(13-35)$ & 0.004 \\
Male (\%) & 22 & 11 & \\
Procedural success (\%) & 89 & 100 & $\mathrm{NS}$ \\
Complication rate (\%) & 11 & 47 & 0.02 \\
Complications affecting management (\%) & 11 & 21 & $\mathrm{NS}$ \\
Decrease RVEDD at six months (\%) & 17.5 & 15.1 & $\mathrm{NS}$ \\
Decrease cardiothoracic ratio at six months (\%) & 7.9 & 7.5 & $\mathrm{NS}$ \\
Median hospital stay (days) & 1 & 6 & $<0.01$ \\
Median cost (£, range) & $5375(5252-8439)$ & $5412(5112-7512)$ & $\mathrm{NS}$ \\
Median time to normal activities (weeks) & 2 & 5.5 & $<0.01$ \\
\hline NS, not significant; RVEDD, right ventricular end diastolic diameter & & \\
\hline
\end{tabular}

\section{Surgical closure}

Surgical closure was carried out using the standard technique under cardiopulmonary bypass by one of two cardiothoracic surgeons using direct suture or autologous pericardial patch.

\section{Complications}

Complications were prospectively recorded and divided into those affecting management and incidental findings. Patients were considered to have a pericardial effusion if there was a global collection greater than $3 \mathrm{~mm}$ on the echocardiogram. All patients with a postprocedural pericardial effusion had increased follow up surveillance until it resolved completely.

\section{Non-invasive measurements}

Non-invasive assessment consisted of end inspiratory chest radiography, standard 12 lead ECG, and transthoracic echocardiogram and was carried out before the procedure, on day 1 , at six months, and at one year postprocedure. Patients after surgical closure of an ASD also had an echocardiogram on the day before discharge. All Amplatzer patients had a transoesophageal echocardiogram at three months after device insertion as part of our institutional protocol. Right ventricular dimensions were measured by transthoracic echocardiography in both groups of patients according to the technique of Ning and colleagues. ${ }^{7}$ Briefly, measurements were taken from the endocardium of the anterior wall of the right ventricle to the septum at the level of the tricuspid annulus in a four chamber apical view at end diastole. Three measurements were taken at the expiratory phase, with the least respiratory variation and the average taken.

\section{Cost}

An institutional accountant calculated cost per case based on information supplied on staff and disposables used, catheter laboratory or theatre time, anaesthetic costs, laboratory or clinical tests, and hospital stay. Patients were questioned about time taken off work or school using a confidential postal questionnaire sent after their discharge home.

\section{Statistical analysis}

Statistical analysis between the two groups was performed using the Mann-Whitney U test and Fisher's exact test. A probability value of $\mathrm{p}<0.05$ was considered significant.

\section{RESULTS}

\section{Patient groups}

Table l summarises the demographic characteristics of the groups, which differed demographically and anatomically. The Amplatzer group was older (median 9.7 years) than the surgical group (median 5.5 years) $(p=0.01)$ and had smaller defects (median $13 v 19 \mathrm{~mm}$ ). These differences are explained by the selected nature of the groups, with the technical limitations of Amplatzer ASD occlusion favouring smaller defects in larger patients.

One patient in the Amplatzer group presented again four years after surgical ASD closure with right ventricular dilatation caused by patch dehiscence. The residual leaks were successfully occluded with two Amplatzer devices.

\section{Procedural success and complications}

There were no deaths in either group. Transcatheter deployment was successful in 24 of 27 (89\%) patients. Three patients had successful Amplatzer ASD closures despite multiple defects that had not been recognised on precordial echocardiography. Device placement failed because a stable device position could not be achieved in two patients. In another an Amplatzer device was successfully deployed but embolised within the first 24 hours to the right ventricle. All three patients underwent successful surgical ASD closure (in two cases some months later) and were included in subsequent surgical analysis. Surgical closure was successful in all 19 patients.

Complications affecting management were observed in 4 of $19(21 \%)$ surgical patients (one had pericardial pain requiring non-steroidal anti inflammatory drugs, one had global pericardial effusion requiring drainage $(250 \mathrm{ml}$ in the first 24 hours), one patient had anaemia requiring haematinics in addition to an incidental pericardial effusion, and one had further incidental pericardial effusion). However, only 10 of the 19 surgical patients (53\%) were completely free from complications. The five remaining complications were minor (small pneumothoraces in three, junctional tachycardia in one, and a small pleural effusion on predischarge chest radiography) and did not require specific treatment.

\section{Non-invasive follow up}

Small leaks around the inferior margin of the device were seen in 3 of 22 patients on early postprocedural scans (on day 1 and transoesophageal echocardiogram at three months). These had all resolved by the six month echocardiogram.

Reduction in right ventricular end diastolic diameter (RVEDD) and cardiothoracic ratio (table 1) are expressed as the percentage change relative to the original preoperative measurements. Two patients in the Amplatzer group were pregnant during follow up and did not have chest radiography. The median reduction in RVEDD and cardiothoracic ratio at six months' follow up was similar for both groups.

The Amplatzer median RVEDD reduction at the six month follow up was $17.5 \%$ (range $0-45.8 \%$ ) and the surgical median RVEDD reduction was $15.1 \%$ (range $0-56 \%)(p=0.15)$ (fig l). The Amplatzer group median cardiothoracic ratio reduction was $7.9 \%$ (range $0-28 \%$ ) compared with a surgical median reduction of $7.5 \%$ (range $0-31 \%)(p=0.56)$. The change in RVEDD in both groups was not predicted by ASD size or patient age. 

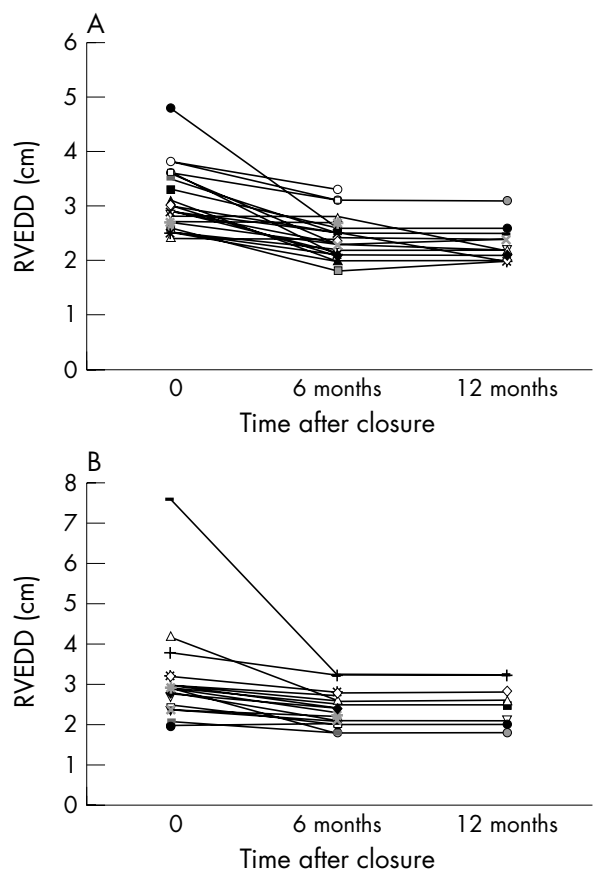

Figure 1 Change in right ventricular end diastolic diameter in $(A)$ the Amplatzer group and $(B)$ the surgical group.

There was a decrease in RVEDD at six months in all but five patients (three non-surgical, two surgical). In one of these patients there was a subsequent decrease in the RVEDD (21\%) at the one year follow up with resolution of paradoxical septal motion. There was no change in the RVEDD in two other patients by 12 months and no 12 month follow up data are available on the remaining two.

\section{Cost and patient stay}

The median inpatient stay for the non-surgical group was one day (range 1-2 days) and for the surgical group six days (range 4-20 days) $(\mathrm{p}<0.01)$. Patients returned to normal daily activities after a median two weeks (range $0.2-26$ weeks) for the Amplatzer group and a median 5.5 weeks (range 4-9 weeks) $(\mathrm{p}<0.01)$ for the surgical group.

The median cost to the institution per successful procedure was $£ 5375$ (range $£ 5252-£ 8349$ ) for the Amplatzer ASD group compared with £5412 (range £5112-£7512) for the surgical group ( $p=N S$ ). The main cost differences were for equipment (median Amplatzer $£ 2974 v$ surgical $£ 408$ ) and inpatient stay (median Amplatzer £300 v surgical £2410).

\section{DISCUSSION}

While it is established that both surgical and transcatheter closure of ASDs carry a low mortality ${ }^{5-12}$ the two techniques have so far been compared only retrospectively. ${ }^{13}$ The aim of our study was to compare prospectively both techniques in detail. We considered effectiveness in terms of procedural success, complications, subsequent regression of right ventricular volume loading, cost, hospital stay, and time to return to normal activities.

Fewer patients undergoing Amplatzer ASD occlusion had a successful initial procedure than those undergoing surgery, in contrast to an earlier report quoting almost complete success rates for both procedures. ${ }^{13}$ The three technical failures in the Amplatzer group (subsequently requiring surgery) and the successful Amplatzer ASD occlusion after surgical dehiscence illustrate the complimentary role for both techniques in patients in whom the primary procedure fails. Most series quote cardiac complication rates of approximately $10-20 \%$ after surgical ASD closure. ${ }^{13-15}$ In the comparative study by Berger and colleagues ${ }^{13}$ there was also no significant difference in the overall subsequent complication rate between surgical and Amplatzer ASD closure. We found a significant difference between the numbers of patients in each group who were affected by at least one complication (11\% and $47 \%$ for non-surgical and surgical closure, respectively, $\mathrm{p}<0.02$ ). However, after excluding minor postsurgical complications that did not affect management (small pneumothoraces, junctional tachycardia), there was no significant difference between the two groups ( $11 \%$ and $21 \%$, respectively, $p=N S$ ). Both techniques were similarly effective in reducing right ventricular dilatation at six months' follow up. There are few data on resolution of right ventricular volume loading after ASD closure. ${ }^{716}{ }^{17}$ Berger and colleagues ${ }^{17}$ describes normalisation within one week of closure, but other authors ${ }^{7}{ }^{16}$ report that ventricular dilatation can persist for up to five years. In our study, we saw almost no change in tricuspid annular diameter in early postprocedural scans ( $<6$ months). Although there was some resolution of right ventricular dilatation by six months, all of the patients were still outside the age corrected normal range for RVEDD. ${ }^{18}{ }^{19}$ Further regression was seen in some of the patients with follow up data at one year but still none of these subjects had normal RVEDD.

Cost analysis shows that the two procedures have similar resource implications in this National Health Service costing framework. Comparison of median cost does not reflect the cost burden of the additional Amplatzer devices used in unsuccessful closure attempts and the two devices used in a single patient. The mean Amplatzer ASD closure cost is $£ 363$ higher than the cost of surgical closure. The main areas of difference between the groups were the high cost of the Amplatzer device and longer hospital stay in the surgical patients. Similar differences in cost break down were reported by Prieto and colleagues ${ }^{20}$ and Gray and associates ${ }^{21}$ in comparisons of surgical with non-surgical persistent ductus arteriosus closure.

Median time to return to normal activities was significantly shorter in the Amplatzer group and much shorter than the 3-14 days reported by Berger and colleagues. ${ }^{13}$ Interestingly, the range of time taken off work or school was wide in both groups, which was often contrary to the advice given to the patients on the timing of return to normal activities.

\section{Conclusions}

Amplatzer ASD closure has a lower chance of success with a single procedure than surgery. Overall there were more complications in the surgical group but the majority of these were minor and did not require any change in management. Resolution of right ventricular dilatation is similar after both types of treatment. Hospital stay and time taken to return to normal activities was significantly shorter for the Amplatzer group patients, but median cost was similar for both groups.

\section{Authors' affiliations}

J D R Thomson, E H Aburawi, K G Watterson, C Van Doorn, J L Gibbs, Department of Paediatric Cardiology and Cardiac Surgery, The Yorkshire Heart Centre, Leeds General Infirmary, Great George St, Leeds LSI 3EX, UK

\section{REFERENCES}

1 King TD, Thompson SL, Steiner C, Mills NL. Secundum atrial septal defects: nonoperative closure during cardiac catheterization. JAMA 1976;235:2506-9.

2 Lock JE, Rome JJ, Davis R, et al. Transcatheter closure of atrial septal defects. Circulation 1989:79:1091-9.

3 Sideris EB, Sideris SE, Thanopoulos BD, et al. Transvenous atrial septal defect occlusion by the buttoned device. Am J Cardiol $1990 ; 66$ : 1524-6.

4 Hausdorf G, Schneider M, Franzbach B, et al. Transcatheter closure of secundum atrial septal defects with the atrial septal defect occlusion system (ASDOS): initial experience in children. Heart 1996;75:83-8. 
5 Chan KC, Godman MJ, Walsh K, et al. Transcatheter closure of atrial septal defect and interatrial communications with a new self expanding nitinol double disc device (Amplatzer septal occluder): multicentre UK experience. Heart 1999;82:300-6.

6 Reddy SC, Rao PS, Ewenko J, et al. Echocardiographic predictors of success of catheter closure of atrial septal defect with the buttoned device. Am Heart J 1995;129:76-82.

7 Ning SB, Fazal H, Cook D. Right ventricular size and ventricular septal motion after repair of atrial septal defect in children. Can J Surg 1984:27:395-8.

8 Murphy JG, Bersh BJ, McGoon MD, et al. Long term outcome after surgical repair of isolated atrial septal defect: follow up at 27 to 32 years. N Engl J Med 1990;323:1645-50.

9 Galal MO, Wobst A, Halees Z. Peri-operative complications following surgical closure of atrial septal defect type II in 232 patients: a baseline study. Eur Heart J 1994;15:1381-4.

10 Kalmar P, Irrgang E. Cardiac surgery in the Federal Republic of Germany during 1990: a report by the German Society for Thoracic and Cardiovascular Surgery. Thorac Cardiovasc Surg 1991;39:167-9.

11 Prieto LR, Foreman CK, Cheatham JP, et al. Intermediate-term outcome of transcatheter secundum atrial septal defect closure using the Bard clamshell septal umbrella. Am J Cardiol 1996;78:1310-2.

12 Berger F, Ewert P, Bjornstad PG, et al. Transcatheter closure as standard treatment for most interatrial defects: experience in 200 patients treated with the Amplatzer septal occluder. Cardiol Young 1999:9:468-73.
13 Berger F, Vogel M, Alexi-Meskishvili V, et al. Comparison of results and complications of surgical and Amplatzer device closure of atrial septal defects. J Thorac Cardiovasc Surg 1999;1 18:674-80

14 Stansel HC Jr, Talner NS, Deren MM, et al. Surgical treatment of atrial septal defect: analysis of 150 corrective operations. Am J Surg 1971;1 21:485-9.

16 Horvath KA, Burke RP, Collins JJ Jr, et al. Surgical treatment of adult atrial septal defect: early and long-term results. J Am Coll Cardiol 1992;20: 1156-9.

16 Meyer RA, Korfhagen JC, Covitz W, et al. Long-term follow-up study after closure of secundum atrial septal defect in children: an echocardiographic study. Am J Cardiol 1982;50:143-8.

17 Berger F, Jin Z, Ishihashi K. Comparison of acute effects on right ventricular haemodynamics of surgical versus interventional closure of atrial septal defects. Cardiol Young 1999;9:484-7.

18 Foale R, Nihoyannopoulos P, McKenna W. Echocardiographic measurement of the normal adult right ventricle. Br Heart J 1986:56:33-44.

19 Gutgesell HP, Paquet M. Atlas of pediatric echocardiography. Gutgesell HP, Paquet $M$. Atlas of pec

20 Prieto LR, DeCamillo DM, Konrad DJ, et al. Comparison of cost and clinical outcome between transcatheter coil occlusion and surgical closure of isolated patent ductus arteriosus. Pediatrics 1998;101:1020-4

21 Gray DT, Fyler DC, Walker AM, et al. Clinical outcomes and costs of transcatheter as compared with surgical closure of patent ductus arteriosus. The patent ductus arteriosus closure comparative study group. N Engl J Med 1993;329:1517-23.

\section{IMAGES IN CARDIOLOGY}

\section{Haemodynamically insignificant fast ventricular tachycardia}

O ur pictures demonstrate one instance in which intractable fast ventricular tachycardia is neither fatal nor haemodynamically significant.

Our patient was a 59 year old man who was considered unsuitable for an acute heart transplant due to multi-organ failure following an extensive anterior myocardial infarction. He was on maximal medical treatment including intra-aortic balloon pump support. Ventricular tachycardias were noted on admission. Following successful implantation of a biventricular assist device (Thoratec, USA) he developed intractable ventricular tachycardia (refractory to antiarrhythmic therapy and DC cardioversion). Despite this usually fatal persistent arrhythmia, he was able to rehabilitate normally. This phenomenon was possible only because of his mechanical biventricular support. This also facilitated sufficient improvement in his haemodynamic, ventilatory, and nutritional status such that he underwent a heart and kidney transplant four months after his initial presentation.

Implantation of ventricular assist devices as a bridge to transplantation or recovery is predominantly for intractable heart failure. Biventricular assist devices account for approximately $20 \%$ of these procedures. Significant ventricular arrhythmias are rare following such operations, but they can result in abnormal pump performance, embolic
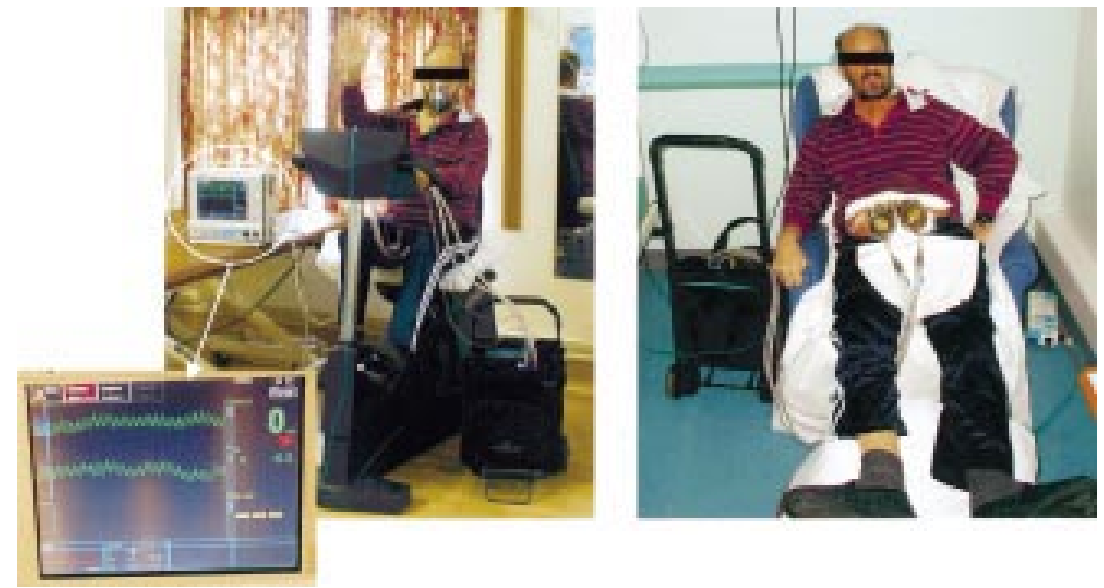

Patient on exercise bicycle (left) and at rest (right). Inset: Underlying rhythm of ventricular tachycardia on ECG monitor. The exposed extra-corporeal ventricular assist devices are seen resting on the patient's abdomen. The portable ventricular device console is present on the left A haemofiltration catheter is visible on the right side of the patient's neck. The motion artefact seen on the monitor in the main picture is abolished in the inset picture taken at rest.

phenomena, and may result in dehiscence of the pump anastomoses. Our patient had none of these complications. We demonstrate the potential of this treatment in selected patients with haemodynamically significant arrhythmias unsuitable for implantable cardioverter-defibrillators.
D K Satchithananda

S Tsui S R Large steven.tsui@papworth-tr.anglox.nhs.uk

We are grateful to the patient's family for allowing publication of this article. 